\title{
ON THE MODELS OF NONLOCAL NONLINEAR OPTICS.*
}

\author{
BORIS G. KONOPELCHENKO AND ANTONIO MORO \\ Dipartimento di Fisica dell'Università di Lecce \\ and Istituto Nazionale di Fisica Nucleare, Sezione di Lecce \\ via Arnesano, I-73100, Lecce \\ E-mail: konopel@le.infn.it, antonio.moro@le.infn.it
}

\begin{abstract}
We show that under certain assumptions a general model of nonlocal nonlinear response in $1+1$-dimension is equivalent to the model considered by Królikowski and Bang for a Kerr-type medium. We derive the limit of weak nonlocality in high frequency regime and discuss the integrable cases.
\end{abstract}

\section{Introduction}

A paraxial laser beam propagating in a medium with response of the form $\mathbf{D}=\varepsilon_{0} \mathbf{E}+\sigma^{3} \mathbf{D}^{(3)}(\sigma<<1)$ is described by the following equation

$$
2 i \sqrt{\varepsilon_{0}} \omega \frac{\partial \mathbf{E}}{\partial z}+\nabla_{\perp}^{2} \mathbf{E}+\omega^{2} \mathbf{D}^{(3)}=0,
$$

where $\mathbf{D}^{(3)}$ is a certain function of the electric field. For derivation of such type of equation see [1,2]. Recent theoretical and experimental results highlight a very interesting phenomenology, such as "accessible solitons", collapse arrest, optical vortices (see e.g. [3-6] and references therein), associated with so-called nonlocal nonlinear media. Most of the models studied before are not integrable and exact analytical solutions are not available for them. However, recently, it was shown that certain choice of nonlocal responses leads to $2+1$-dimensional integrable models ${ }^{7,8}$.

In the present paper, for sake of simplicity we discuss the $1+$ 1 -dimensional case. In the section 2 , we consider a more fundamental nonlocal nonlinear model than one considered in the paper [7]. We specify under which conditions it leads us to a generalization of the model discussed by Królikowski and Bang ${ }^{9}$. The model proposed in [9] is of interest since

*This work is partially supported by COFIN PRIN SINTESI 2004. 
it can be solved analytically for weak nonlocality and it admits soliton-like solutions.

In the section 3 we analyze a class of weak nonlocal responses in the high frequency regime. The study of high frequency limit can help to detect interesting properties of the beam such as singular phases and consequent vortex type behaviours. Moreover, this study could be useful to construct new nontrivial ansatz for applying a variational method. Looking for solutions which depend "slowly" on the coordinate along the propagating direction, we show that the phase of the electric field is given by an overdetermined system of partial differential equations for the phase. Their compatibility and some integrable cases are also discussed.

\section{The nonlocal nonlinear Schrödinger equation.}

Equation (1) in 1+1-dimensional case looks like as follows

$$
2 i \omega \frac{\partial \mathbf{E}}{\partial z}+\frac{\partial^{2} \mathbf{E}}{\partial x^{2}}+\omega^{2} \mathbf{D}^{(3)}=0 .
$$

We set $\varepsilon_{0}=1$ without lost of generality. Let us assume a constitutive relation of the following general form

$$
\mathbf{D}^{(3)}=\int_{-\infty}^{+\infty} R\left(x-x^{\prime} ; a\right) N\left(I\left(x^{\prime}\right)\right) \mathbf{E}\left(x^{\prime}\right) d x^{\prime} .
$$

Distribution $R\left(x-x^{\prime} ; a\right)$ characterizes the nonlocal response around the point $x$ and $a$ is the "width" parameter (in the following it will be assumed to be depending on the frequency $\omega$ ). $N(I)$ is an arbitrary nonlinear response depending on the intensity of the electric field $I=|\mathbf{E}|^{2}$. In what follows we will propose a way to simplify the general model (3) which will lead us to model considered in the paper [9].

Let us consider a nonlocal distribution $R\left(x-x^{\prime} ; a\right)$ of width $\delta R$ defined as the minimum such that

$$
R\left(x-x^{\prime} ; a\right) \simeq 0, \quad \forall x^{\prime} \notin[x-\delta R, x+\delta R] .
$$

Analogously we introduce the widths $\delta E$ and $\delta N$ of the electric field and the nonlinear response respectively. Suppose they verify the following conditions

$$
\delta R \sim \delta N, \quad \delta R<<\delta E .
$$

Let us assume a nonlinear response of the form

$$
N(I(x))=\tilde{N}(X),
$$


where $X=\gamma x$ and $\gamma=1 / \delta N$. Expanding $\mathbf{E}\left(x^{\prime}\right)$ and $N\left(I\left(x^{\prime}\right)\right)$ in Taylor series around $x$, one gets the following approximation of the formula (3)

$$
\mathbf{D}^{(3)} \simeq\left(\int_{-\infty}^{+\infty} R\left(x-x^{\prime} ; a\right) N\left(I\left(x^{\prime}\right)\right) d x^{\prime}\right) \mathbf{E}(x),
$$

where we kept into account that due to the equation (6) higher orders of the expansion of $N(I(x))$ are not negligible. Note that the formula (7), in the case of nonlocal Kerr-type medium, leads to the nonlocal nonlinear Schrödinger equation discussed in the paper [9].

For instance, given a bell-shape electric field $\mathbf{E}=\mathbf{E}_{0} \exp \left[-x^{2} / 2 \sigma^{2}\right]$, a nonlinear response of the form $N(I)=I^{\alpha}=\left|\mathbf{E}_{0}\right|^{2 \alpha} \exp \left[-(\gamma x)^{2} / 2 \sigma^{2}\right]$, where $\gamma=\sqrt{2 \alpha}$, satisfies the condition (6). Nevertheless, it's easy to see that validity of relations (5) is sufficient to obtain the model (7). For instance, choosing $\mathbf{E}=\mathbf{E}_{0} / \cosh ^{2}(x)$ and $N(I)=I^{\alpha}$, condition (5) is verified for $\alpha$ large enough.

\section{Weak nonlocality and high frequency limit.}

Let us consider a limit of small nonlocality $(\delta R<<1)$ such that it is reasonable to expand both $\mathbf{E}$ and $N$ in Taylor series. This expansion gives

$$
\begin{aligned}
\mathbf{D}= & N(I(x)) \mathbf{E}(x)+R_{1}\left(N \frac{\partial \mathbf{E}}{\partial x}+\frac{\partial N}{\partial x} \mathbf{E}\right)+ \\
& R_{2}\left(\frac{1}{2} N \frac{\partial^{2} \mathbf{E}}{\partial x^{2}}+\frac{\partial N}{\partial x} \frac{\partial \mathbf{E}}{\partial x}+\frac{1}{2} \frac{\partial^{2} N}{\partial x^{2}} \mathbf{E}\right)+ \\
& R_{3}\left(\frac{1}{6} N \frac{\partial^{3} \mathbf{E}}{\partial x^{3}}+\frac{1}{2} \frac{\partial N}{\partial x} \frac{\partial^{2} \mathbf{E}}{\partial x^{2}}+\frac{1}{2} \frac{\partial^{2} N}{\partial x^{2}} \frac{\partial \mathbf{E}}{\partial x}+\frac{1}{6} \frac{\partial^{3} N}{\partial x^{3}} \mathbf{E}\right)+\ldots
\end{aligned}
$$

where the distribution $R\left(x-x^{\prime} ; a\right)$ is assumed to be normalized

$$
\int_{-\infty}^{+\infty} R\left(x-x^{\prime} ; a\right) d x^{\prime}=1
$$

and $R_{n}$ are the $n$-moments

$$
R_{n}=\int_{-\infty}^{+\infty} R\left(x-x^{\prime} ; a\right)\left(x-x^{\prime}\right)^{n} d x^{\prime} .
$$

In the present section, we are interested to perform the high frequency limit. In particular, we assume that for $\omega \rightarrow \infty$ the effective nonlocality decreases because of rapid oscillation of the electric field. So, one has

$$
\lim _{\omega \rightarrow \infty} R\left(x-x^{\prime} ; a(\omega)\right)=\delta\left(x-x^{\prime}\right),
$$


where the parameter $a$ is a function of the frequency such that there exists its limit for $\omega \rightarrow \infty$ and $\delta\left(x-x^{\prime}\right)$ is the Dirac $\delta$-function.

As illustrative example, we focus on the distribution defined as follows

$$
R\left(x-x^{\prime} ; a\right)=\left\{\begin{array}{rr}
\frac{3}{4}\left(-a^{3}\left(x-x^{\prime}\right)^{2}+a\right) & x^{\prime} \in\left[x-\frac{1}{a}, x+\frac{1}{a}\right] \\
0 & x^{\prime} \notin\left[x-\frac{1}{a}, x+\frac{1}{a}\right]
\end{array}\right.
$$

It is straightforward to verify that distribution (12) tends to the $\delta$-function as $a \rightarrow \infty$, that is

$$
\begin{aligned}
\int_{-\infty}^{\infty} R\left(x-x^{\prime} ; a\right) d x^{\prime} & =1 \\
\lim _{a \rightarrow \infty} \int_{-\infty}^{+\infty} R\left(x-x^{\prime} ; a\right) f\left(x^{\prime}\right) d x^{\prime} & =f(x) .
\end{aligned}
$$

A direct calculus shows that

$$
R_{2 n}=\frac{r_{2 n}}{a^{2 n}}, \quad R_{2 n+1}=0, \quad n \in \mathbb{N} \cup\{0\} .
$$

We would like to stress that the present discussion still holds for any nonlocal response whose moments are of the form (14).

Let us consider a general dispersion law of the following form

$$
N=N_{0}+\frac{\tilde{N}}{1+\left(i \tau_{0} \omega\right)^{2 \nu}},
$$

where $\tau_{0}$ is usually called relaxation time. In the high frequency limit $\omega \rightarrow \infty$, expanding $I=I_{0}+\omega^{-2 \nu} I_{1}+\ldots$, one gets

$$
N=N_{0}\left(I_{0}\right)+\frac{N_{1}\left(I_{0}, I_{1}\right)}{\omega^{2 \nu}}+O\left(\frac{1}{\omega^{4 \nu}}\right),
$$

where $N_{1}$ is complex-valued and $2 \nu>0$. For $2 \nu<1$, relation (15) is known as Cole-Cole dispersion law ${ }^{10}$ which substitutes the "classical" Debye law $(2 \nu=1)$ for a wide class of liquid and solid polar media.

We perform the high frequency limit in usual way looking for solutions of the form

$$
\mathbf{E}=\mathbf{E}_{0} e^{i \omega S} .
$$

Moreover, we assume that the electric field depends slowly on the $z$-variable according to the rule

$$
\frac{\partial}{\partial z} \rightarrow \omega^{-2 \nu} \frac{\partial}{\partial z} .
$$


It is straightforward to see that under the assumption given above and under the choice $\alpha=1+\nu$, the high frequency limit of equation (2) leads, at $\omega^{2}$ and $\omega^{2-2 \nu}$ orders, to the following system

$$
\begin{aligned}
S_{x}^{2} & =N_{0} \\
S_{z} & =-\frac{r_{2}}{4} N_{0} S_{x}^{2}+\frac{1}{2} N_{1},
\end{aligned}
$$

where $f_{x}=\partial f / \partial x$.

System (19) is over-determined and its compatibility condition is equivalent to the following nonlinear equation

$$
\pm N_{0}^{-\frac{1}{2}} N_{0 z}=-r_{2} N_{0} N_{0 x}+N_{1 x} .
$$

For $N_{1} \neq 0$ solutions of equation (20) are complex-valued, and they implies complex-valued phases. Such solutions are connected in principle with absorption or amplification of the electric field.

We note that, once $R$ is assigned, the model (7), at the order $\omega^{2-2 \nu}$, provides us with the equation

$$
S_{z}=\frac{1}{2} N_{1},
$$

and the compatibility condition can be written down in the form of conservation law $M_{0 z}=N_{1 x}$ where $M_{0}= \pm \sqrt{N_{0}}$.

If we assume $N_{1}=0$, setting $N_{0}=\varphi^{\frac{2}{3}}$ equation (20) is reduced to the well known Burgers-Hopf equation

$$
\varphi_{z}= \pm \varphi \varphi_{x}
$$

It is solvable with the hodograph method and the solutions are given in terms of the following implicit relation

$$
x \pm \varphi z+H(\varphi)=0,
$$

where $H$ is an arbitrary function of its argument.

Now, let us assume $N_{1}=N_{1}\left(N_{0}\right)$. Even in this case, equation (20) is reduced to the following quasilinear equation

$$
N_{0 z}= \pm N_{0}^{\frac{1}{2}}\left(N_{1}^{\prime}\left(N_{0}\right)-r_{2} N_{0}\right) N_{0 x},
$$

where $N_{1}^{\prime}=d N_{1} / d N_{0}$.

Analogously to the Burgers-Hopf equation it is solved by the hodograph relation

$$
x \pm N_{0}^{\frac{1}{2}}\left(N_{1}^{\prime}\left(N_{0}\right)-r_{2} N_{0}\right) z+L\left(N_{0}\right)=0,
$$


where $L$ is an arbitrary function of $N_{0}$.

Other integrable equations can be obtained considering nonlocal response of the following form

$$
R\left(x-x^{\prime} ; a\right)=\left\{\begin{aligned}
\frac{n+1}{1-(-1)^{n}+2 n}\left(-a^{n+1}\left(x-x^{\prime}\right)^{n}+a\right), & x^{\prime} \in D \\
0, & x^{\prime} \notin D
\end{aligned}\right.
$$

where $n \in \mathbb{N}$ and $D=\left[x-\frac{1}{a}, x+\frac{1}{a}\right]$. In this case, the $n$-moments are

$$
R_{m}=\frac{1-(-1)^{m}}{2} \frac{r_{m}}{a^{m}} .
$$

For any even $n$ one gets the system (19). If $n$ is odd, setting $\alpha=1+2 \nu$ we obtain the system

$$
\begin{aligned}
S_{x}^{2} & =N_{0} \\
S_{z} & =\frac{i r_{1}}{2} N_{0} S_{x}+\frac{1}{2} N_{1} .
\end{aligned}
$$

Compatibility condition is equivalent to the equation

$$
N_{0 z}=i \frac{3}{2} r_{1} N_{0} N_{0 x} \pm \sqrt{N_{0}} N_{1 x} .
$$

\section{Acknowledgments}

A.M. is pleased to thank Prof. W. Królikowski for useful discussions.

\section{References}

1. L.D. Landau, E.M. Lifshitz and L.P. Pitaevski, Electrodynamics of continuous media vol.8, Pergamon Press (1984).

2. C. Sulem and P. Sulem, The nonlinear Schrödinger equation, Springer-VerlagBerlin (1999).

3. A.W. Snyder and D.J. Mitchell, Science 276, 1538 (1997).

4. C. Conti, M. Peccianti and G. Assanto, Phys. Rev. Lett. 92, 113902 (2004).

5. O. Bang, W. Królikowski, J. Wyller and J.J. Rasmussen, Phys. Rev. E 66, 046619 (2002).

6. D. Briedis, D.E. Petersen, D. Edmundson, W. Królikowski, O. Bang, Optics Express 13(2), 435 (2005).

7. B. Konopelchenko and A. Moro, Paraxial light in a Cole-Cole nonlocal medium: integrable regimes and singularities, Proc. of "SPIE Int. Conf. on Optics and Optoelectronics" (August 28 - September 03, 2005, Warsaw) vol. 5949. Preprint arXiv:nlin.SI/0506012 (2005).

8. B. Konopelchenko and A. Moro, Stud. Appl. Math. 113, 325 (2004).

9. W. Królikowski and O. Bang, Phys. Rev. E 63, 016610 (2000).

10. K.S. Cole and R.H. Cole, J. Chem. Phys. 9, 341 (1941). 